\title{
Beam weapons still science fiction
}

\section{- US physicists deliver sceptical report - SDI timetable is threatened}

Washington

A DECADE of research is needed even to decide if laser and particle beam weapons are capable of performing the crucial tasks that proponents of the Strategic Defense Initiative (SDI) envisage for them. That is the conclusion of an American Physical Society (APS) report, released last Thursday after a long gestation. In the most thorough examination so far of directed energy weapons, a seventeen-member panel of leading US physicists has concluded that all proposed technologies fall far short of the performance that SDI would demand.

The report has been twenty-one months in the making, the last seven taken up with classification reviews by the Department of Defense (DOD). The panel had access to classified material, so that an authoritative report could be compiled, but public release was then dependent on DOD scrutiny. The amount of material deleted was "minuscule", according to Kumar Patel, research director at Bell Laboratories and co-chairman of the panel with Nicolaas Bloembergen of Harvard University. The most significant omissions in the final report concern the vulnerability of the defensive systems and possible Soviet counter-measures.

Although the report does not address general questions of battle management and computer software complexity, it is much more than a technical assessment of tested ideas. barely understood. individual weapons. If the launch of ballistic missiles is accompanied by a much greater number of decoys, the defence has a complex task: it must detect all fastmoving objects (giving only a few false alarms in so doing), distinguish missiles from decoys, track each missile with microradian accuracy, direct an intense, tightly focused beam precisely onto a target, make sure that a kill has been made, and then retarget the beam quickly onto the next missile. In all these facets of operation, and especially in discriminating between missiles and decoys, the report finds many vague concepts and few

SDI research has produced some working devices. A technical summary is in the accompanying inset, but the basic conclusion is that improvement by two orders of magnitude or more in power, for focusing and accuracy is needed for any of today's techniques to find military application. Furthermore, scaling up present devices is much more than a technicality; in many cases fundamental principles are still

But the DOD is not put out. In an official response they describe the report as "subjective and unduly pessimistic", and they claim significant progress during the seven months that the report was going through review. They point to a new injector for free-electron lasers at Los Alamos Laboratory, and a high power,

\section{Where the beam-weapon technologies stand:}

- Chemical lasers based on hydrogen or deuterium fluoride are the most advanced candidate, but their energy output would have to be increased at least $\mathbf{1 0 0}$-fold while maintaining beam quality. Other chemical lasers, such as the atomic iodine type, need to be scaled up by much larger factors.

- Excimer lasers fall short by four orders of magnitude in power, and their pulse repetition rate is much too low.

- Free-electron lasers, newer and less well understood, are so far almost a million times too feeble.

- X-ray lasers pumped by nuclear explosions have been much touted as the answer to SDI's problems, but only a single underground test has been conducted, and the feasibility of a working weapon is entirely undemonstrated

- Neutral particle beam weapons show some promise, but continuous high current beams seem hard to achieve. Neutral particle weapons would have to operate above the atmosphere, and techniques for repeatedly and accurately aiming the beam have yet to be devised.

- Electron beams can perhaps be shot along plasma channels punched through the atmosphere by a laser; this prevents the terrestrial magnetic field from deflecting the beams. But the creation of plasma channels is largely unproved, and the electron beams must be scaled up in voltage, pulse duration and average power. - Propagation of intense, tightly focused optical laser beams presents many problems. A groundbased system would have to allow for atmospheric aberrations, and for the weather (with seven stations in the US, there would on average still be one day a year when all are clouded over). Even in space, novel phase correction methods must be used to keep the beam width down to a diffraction-limited focus.

- Detection and tracking of missiles must be almost perfectly reliable. All missiles must be found, and very few false alarms must arise. If missiles are to be tracked during the boost phase by their infrared emisson, then ways must be found of locating the body of the missile within the more extensive hot plume. Accurate tracking of missiles during their midcourse, when they are much cooler, is harder still. Long-wavelength infrared telescopes would have to be at least of $10-\mathrm{m}$ aperture to achieve sufficient resolution. Alternative methods, such as microwave or optical radar, would require numerous space platforms. continuous ion source for neutral beam machines, developed at Culham Laboratory in the United Kingdom. However, panel member Andrew Sessler, former director of Lawrence Berkeley Laboratory, said that although these improvements were significant, they concerned only small parts of big devices, and the report's conclusions were by no means invalidated.

The DOD also stresses that it regards a directed energy weapons system as the second phase of SDI, with kinetic energy weapons, which were not studied by the APS panel, constituting the first phase. The APS report puts a decision just on the

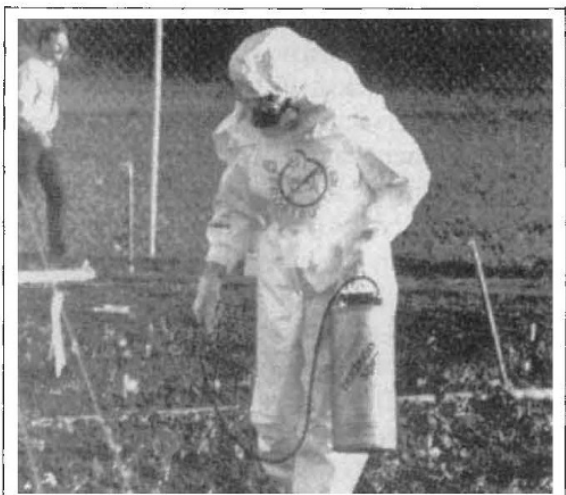

What the well dressed California strawberry grower is wearing this season. See p.819.

feasibility of this second phase at least ten years away and, as Jeremiah Sullivan of the University of Illinois emphasized, the whole idea might well turn out to be unworkable. But Lt-Gen. James Abrahamson, of the Strategic Defense Initiative Office, still believes that continued research at current levels will allow a decision to be made in the early $1990 \mathrm{~s}$, if not before.

Even if official optimism can overcome the report's sober conclusions, a further revelation contained in the report is bound to increase public and international opposition to SDI. Estimates of the power required for space-based systems show that even for 'standby' operation, each platform must have its own nuclear reactor. The prospects of hundreds of reactors being launched into space cuts deeply into SDI's image as a "nonnuclear" defence shield. Even worse, there is talk of plans to use nuclear explosions in space to power a "nuclear shotgun'. Hypervelocity pellets powered by a bomb would rip through space, destroying lightweight decoys and leaving warheads to be picked off by other weapons. The system, codenamed Prometheus, is classified, but is thought to have undergone preliminary testing in an underground nuclear explosion.

David Lindley \& Alun Anderson 